\title{
KEKB CONTROL SYSTEM: THE PRESENT AND THE FUTURE
}

\author{
A. Akiyama, S. Araki, J-I. Odagiri, T. Katoh, T. Kawamoto, \\ I. Komada, K. Kudo, T. T. Nakamura, T. Naito, N. Yamamoto*, \\ KEK, Tsukuba, JAPAN \\ M. Kaji, Mitsubishi Electric Co., Ltd., JAPAN \\ T. Kitabayashi, K. Yoshii, \\ Mitsubishi Electric System \& Service Engineering Co., Ltd., JAPAN \\ N. Koizumi, Mitsubishi Space Software Co., Ltd. JAPAN \\ M. Takagi, S. Yoshida, Kanto Information Service, JAPAN
}

\begin{abstract}
Achievements of KEKB control system during the commissioning of KEKB accelerators will be reported. The KEKB control system is the first major application of EPICS toolkit in JAPAN. We added some software components on the top of EPICS toolkit. One of these is the system to automate generation of the EPICS database configuration files using ORACLE database management system. Although it costs us a lot of effort to develop, this kind of configuration management system is essential for a large accelerator like KEKB. Another component is an implementation of EPICS CA(Channel Access) interface in the script languages. SAD, a modeling program with a scripting language capability, and Python, an object oriented scripting language are currently supported in the KEKB control system. These scripting languages are used to develop high level applications, such as an orbit correction program with graphical user interface using Tk library. Combination of these scripting languages and EPICS CA provides flexibility in the control system. KEKB control system also includes some new hardware components, including Arcnet based magnet power supply controllers and VME single board computers based on Motrolla's PowerPC750 CPU. The performance of these components will also be reported. The problems and (possible) solutions found during the KEKB commissioning will also be discussed.
\end{abstract}

\section{OVERVIEW OF KEKB CONTROL SYSTEM}

Commissioning of KEKB[1], an asymmetric electronpositron collider for B-meson physics, has started on December 1st, 1998 after 5 years of construction. The control system for KEKB accelerators[2] also started its operation shortly before December 1st. Although some applications in the control system had limited functionality, basic operation of the equipment could be performed using KEKB control system.

Network: The KEKB control system includes 26 local control rooms along $3 \mathrm{~km}$ circumference ring accelerators and a central control room. These control rooms are linked

\footnotetext{
*Email: noboru.yamamoto@kek.jp
}



Figure 1: Schematic view of the KEKB control system

Table 1: VME single board computers used in the KEKB control system

\begin{tabular}{|c|c|}
\hline CPU Type & Number of CPU \\
\hline Force PowerCore6750 & 78 \\
\hline Force PowerCore6603 & 13 \\
\hline Force CPU40 & 9 \\
\hline
\end{tabular}

with switched FDDI network [See Figure 1.] In each local control rooms, a VME single board computer, called Input/Output Controller or IOC in EPICS terminology, connected to the KEKB control system network through FDDI-Ethernet bridge. A console port of IOC and a serial port on a system monitor board in each VME subrack are connected to a terminal server and remotely accessible.

IOC: We use VME single-board computers equipped with Motrolla PowerPC 750 (Force PowerCore 6750) as IOCs. We also use some VME single board computers with PowerPC603e and MC68040. 100 IOCs in total are used in the KEKB control system currently.

Fieldbus interfaces: We use several fieldbuses, CAMAC, GP-IB, Arcnet, Modbus+, MXI and the serial line, in the KEKB control system. IOC controls devices on these fieldbuses using a fieldbus interface board in a VME subrack. We developed drivers for Arcnet and Modbus+ inter- 
Table 2: Parameters of the Host computer for KEKB control system

\begin{tabular}{|c|c|c|}
\hline CPU & HP PA-RISC & Alpha \\
\hline \# of CPUs & $4(@ 120 \mathrm{MHz})$ & $\begin{array}{c}4(@ 440 \mathrm{MHz}) \\
\& 6(@ 330 \mathrm{MHz})\end{array}$ \\
\hline memory & 2GB & 1GB \\
\hline Network & FDDI & FDDI \\
\hline Storage & 20GB RAID & 50GB RAID \\
\hline
\end{tabular}

\section{System OverView (cont'd)}

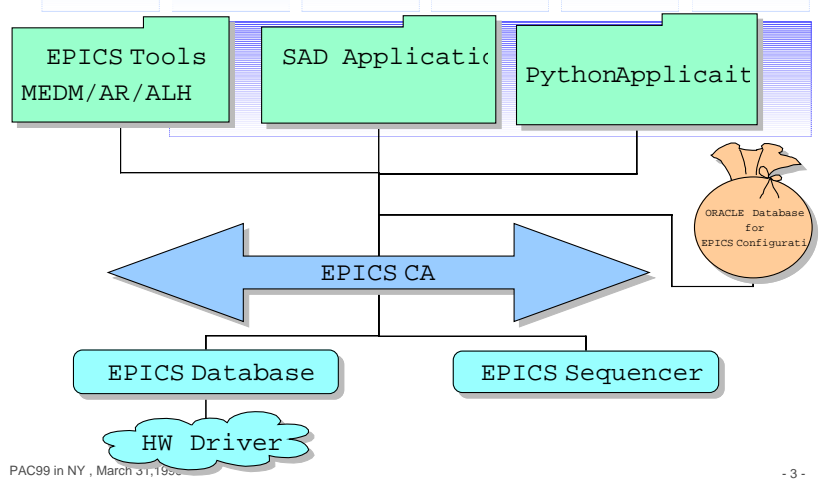

Figure 2: Software components in the KEKB control system

faces. EPICS drivers are used for other fieldbuses.

Host computers: Mitsubishi ME/RK-460 computer(HPUX 10.20) equipped with four CPUs is used as the host computer in the KEKB accelerator control system. The host computer also serves a development environment for VxWorks which is the operating system of IOCs. Xterminals are used as an operator interface in the KEKB accelerator control system.

A cluster of Alpha servers is used as a servers for accelerator modeling/simulation. These servers also have network interfaces to KEKB control network and runs some KEKB control system applications.

Software: Software in the KEKB control system is constructed using the framework of EPICS. PL/SQL programs generates parameter files to generate EPICS database files combined with template databases. MEDM, a GUI tool in EPICS, is used to build a simple user interface. Other applications which has GUI are developed using ether SAD or Python. SAD is an accelerator modeling/simulation program developed at KEK. User of SAD can control the program using SAD script, which has a syntax similar to the syntax in Mathematica[11]

Python is an object oriented interpreter language developed by G. von Rossum [9, 10] Both languages have interfaces to EPICS-CA and Tk widgets and used to build an application which may not fit in MEDM.
Table 3: Some system parameters shows working condition of a host computer in the KEKB control system

\begin{tabular}{rlcccc}
\hline Load average: & $4-5$ & $(4$ & CPU & configuration $)$ \\
Users: & 150 & & & & \\
Processes: & 900 & & & \\
Memory: & AVAIL & USED & FREE & USED \\
Usage(KB): & $1,635,272$ & 275,920 & $1,359,352$ & $17 \%$ \\
\hline
\end{tabular}

\begin{tabular}{|c|c|c|}
\hline 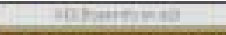 & & 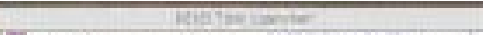 \\
\hline FHKB OFERATION & & $n=1-2$ \\
\hline unsm & & : \\
\hline General I 1 & 17 & $\lg \pi$ \\
\hline cons: & & ; give \\
\hline 표로 & $\bar{z}$ & : gien \\
\hline Nom nese & Ex: & Tenorownos \\
\hline 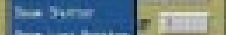 & 貍 & 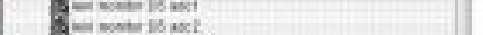 \\
\hline & & $g=\operatorname{sen} i 11 \mathrm{~km}$ \\
\hline 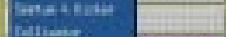 & in & 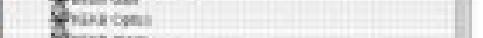 \\
\hline Gents & 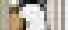 & 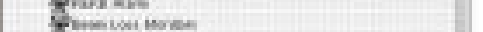 \\
\hline Thates & & 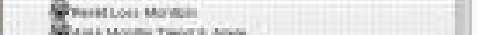 \\
\hline Saling The in & & 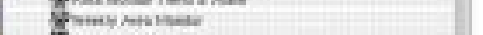 \\
\hline conted & P & Guincun- \\
\hline Operation lis.: & & 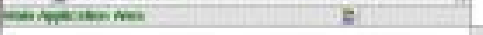 \\
\hline
\end{tabular}

Figure 3: KEKB control system currently has two starts points, KEKB task launcher(right) and KEKBOPERATION.adl(left).

\section{SYSTEM PERFORMANCE}

The Basic unit of information in EPICS is a record. Distributed EPICS runtime database on IOCs keeps 208,716 records in it. Average number of records on an IOC is 2,319. Software and Database on IOC occupy 5MB of memory on average.

In the KKEB control system network, 2000 packets are transfered every second on average.

Table 3 shows some system parameters in working conditions. When we started commissioning of KEKB in last December, the host computer just had two CPUs. "rup" value reached more than 10 and it resulted slow response of the system.

Fieldbus interfaces: 2517 magnet power supplies have Arcnet interface controller board developed at KEK. These interface board are connected to one of 176 Arcnet network segments. 12 IOCs are are used to control magnet power supplies. One IOC controllers up to 200 power supplies and receives a status packet information to IOCs every 5 seconds. Occasional Arcnet network reconfiguration does not harm operation of magnet power supplies.

Software: Figure 3 shows a main screen of KEKB control system. A user or an operator can choose a control application from this screen. More than 120 programs in SAD or Python and 50 MEDM screens are registered to the main control. 


\section{PROBLEMS}

Slow response: When we just started the operation KEKB control system we found some problems in the control system. Most noticeable is slow response of GUI written in script languages. Tuning of Unix system parameters on Unix host and improved situation. Finally we increased number of CPUs on the host computer from 2 to 4 . It drastically improved the situation.

Software collision: Each software components is developed and tested separately and integrated onto a IOC later. This approach works for most of cases but caused incompatibility problems in some cases. In some cases, we took a simple solution, introducing new IOCS and run these applications in separate IOCs.

Console port monitoring: To analyze cause of IOC crashes or suspended vxWorks tasks, we need to correct some data when it occurs. A console port of each IOCs is connected to a port of a terminal servers. A xterm window for each console port is opened on the console for IOC monitoring. It allows us to store messages printed on the console to the file on the host computer and to analyze it later. We also developed simple application to find these information easily.

\section{FUTURE}

Here we list up possible upgrade plans for KEKB control system. Some of them can be installed soon.

EPICS core software upgrade: Currently we use the release 3.13 beta11 of EPICS core software. We should move to the latest release 3.13.1 as soon as possible. Merging of local changes into 3.13.1 is underway. The release 3.13.1 will be used in the KEKB operation after we test drivers and device support routines in the release 3.13.1 environment. This has to be done as soon as possible.

Arcnet upgrade: The current daisy chain configuration of Arcnet will be partly replaced by the star configuration using Arcnet HUB. This change will reduce the chance of network reconfiguration and increase reliability of Arcnet communication.

Dual host computers: Although we use 3 Unix servers for KEKB operation, ORACLE runs only on one of them. Dual server configuration of ORACLE servers is essential for the stable operation of KEKB control system.

network upgrade plan: Because of scalable architecture, it is possible to increase system POWER gradually in principle. However, network bandwidth at the network interfaces on host servers can be a bottleneck of the system in (near) future.

When we designed KEKB control system, FDDI was a mature technology and seemed to have a future upgrade path. ATM was a growing network technology at that time but still is not a standard network technology. 100Base-T and Giga-bit Ethernet technology are gaining the position of de facto standard for future network technology. We have to find smooth way to change network infrastructure .

\section{CONCLUSION}

The KEKB control system has been operational since last December. It is the first large scale implementation of EPICS in JAPAN.

We found that the use of interpreter in an accelerator control system is quite useful. For the accelerator like KEKB, flexibility of the control application is as important as its stability, especially in its commissioning phase.

\section{REFERENCES}

[1] “KEKB B-Factory Design Report”, KEK Report 95-7, August 1995

[2] T. Katoh et al., "Present Status of the KEKB Control System”, ICALEPCS ‘97, Beijing, China, November 3-7, 1997

[3] W. McDowell et al.:"EPICS Home Page", "http://epics.aps.anl.gov/asd/controls/epics/EpicsDocumentation/EpicsGeneral/"

[4] L. Dalesio et al. "Distributed Software Development in the EPICS Collaboration,”, Proc. ICALEPCS95, Chicago, USA, 1995; L. Dalesio et al.: The Experimental Physics and Industrial Control System Architecture: Past, Present, and Future, Proc. ICALEPCS, Berlin, Germany, 1993, pp 179-184.

[5] A. Akiyama et al., "KEKB Power Supply Interface Controller Module”, ICALEPCS '97, Beijing, China, November 3-7, 1997

[6] 'SAD home page' at "http://www-acctheory.kek.jp/SAD/sad.html"

[7] M. Kaji et al., "RELATIONAL DATABASE SYSTEM IN THE KEKB ACCELERATOR CONTROL SYSTEM”, Proc. of the first Asian particle accelerator conference(APAC98), Tsukuba, JAPAN, 1998.

[8] J. Odagiri et al., "Performance Evaluation of EPICS on PowerPC “, ICALEPCS ‘97, Beijing, China, November 3-7, 1997

[9] M. Lutz, "Programming Python", O’Reilly \& Associates, Inc. USA, 1996

[10] 'Python Home Page', "http://www.python.org/"

[11] S. Wolfram, "Mathematica: A system for Doing mathematics by Computer", 2nd ed. Addison-Wesley Publishing Company, Inc. USA, 1991 